\title{
Silicon Waveguide with Lateral p-i-n Diode for Nonlinearity Compensation by On-Chip Optical Phase Conjugation
}

Gajda, A.; Da Ros, Francesco; Porto da Silva, Edson; Pczek, A. ; Liebig, E.; Mai, A.; Galili, Michael; Oxenløwe, Leif Katsuo; Petermann, Klaus; Zimmermann, L.

Published in:

2018 Optical Fiber Communication Conference

Link to article, DOI:

10.1364/OFC.2018.W3E.4

Publication date:

2018

Document Version

Peer reviewed version

Link back to DTU Orbit

Citation (APA):

Gajda, A., Da Ros, F., Porto da Silva, E., Pczek, A., Liebig, E., Mai, A., Galili, M., Oxenløwe, L. K., Petermann, K., \& Zimmermann, L. (2018). Silicon Waveguide with Lateral $p$-i-n Diode for Nonlinearity Compensation by OnChip Optical Phase Conjugation. In 2018 Optical Fiber Communication Conference [Paper W3E.4] Optical Society of America. https://doi.org/10.1364/OFC.2018.W3E.4

\section{General rights}

Copyright and moral rights for the publications made accessible in the public portal are retained by the authors and/or other copyright owners and it is a condition of accessing publications that users recognise and abide by the legal requirements associated with these rights.

- Users may download and print one copy of any publication from the public portal for the purpose of private study or research.

- You may not further distribute the material or use it for any profit-making activity or commercial gain

- You may freely distribute the URL identifying the publication in the public portal 


\title{
Silicon Waveguide with Lateral p-i-n Diode for Nonlinearity Compensation by On-Chip Optical Phase Conjugation
}

\author{
A.Gajda1 ${ }^{1}$, F. Da Ros ${ }^{2}$, E.P. da Silva² ${ }^{2}$ A. Pęczek ${ }^{3}$, E.Liebig ${ }^{4}$, A. Mai ${ }^{1}$, M.Galili², \\ L.K. Oxenløwe ${ }^{2}$, K. Petermann ${ }^{4}$, L.Zimmermann ${ }^{1,4}$ \\ ${ }^{1}$ IHP GmbH, Im Technologiepark 25, 15236 Frankfurt (Oder), Germany, gajda@ihp-microelectronics.com \\ ${ }^{2}$ DTU Fotonik, Technical University of Denmark, DK-2800 Kongens Lyngby, Denmark, fdro@fotonik.dtu.dk \\ ${ }^{3}$ IHP Solutions GmbH, Im Technologiepark 25, 15236 Frankfurt (Oder), Germany \\ ${ }^{4}$ Institut für Hochfrequenz- und Halbleiter-Systemtechnologien, TU Berlin, Einsteinufer 25, Berlin Germany
}

\begin{abstract}
A 1-dB Q-factor improvement through optical phase conjugation in a silicon waveguide with a lateral p-i-n diode enables BER < HD-FEC after 644-km dispersion-compensated transmission for all channels of a 5xWDM 16-QAM single-polarization signal. (C) 2018 The Author(s)

OCIS codes: (190.4380) Nonlinear optics, four-wave mixing, (060.0060) Fiber optics and optical communications,
\end{abstract}

\section{Introduction}

The need for achieving higher spectral efficiencies and data rates driven by the growth of bandwidth demand pushes the limit of a transmission system against the barrier posed by Kerr nonlinearity. In order to maintain the high optical signal-to-noise ratio (OSNR) required by a high-order modulation formats throughout the transmission, higher launch power is required, with the consequent onset of nonlinear signal distortion caused by the Kerr effect in the transmission fiber. Both digital and optical techniques have been investigated to either compensate or mitigate such a distortions. While digital techniques such as digital backpropagation and perturbation-based techniques provide unquestionable benefits, the requirements on the transceiver bandwidth limits their practical use to single-channel operation. Alternatively, all-optical approaches, such as optical phase conjugation (OPC), are more suited to tackle inter-channel interaction. Significant benefits of OPC on improving system performance have been reported in a number of demonstrations in the past years [1-6]. These demonstrations have mainly relied on providing OPC through either four-wave mixing (FWM) in a highly nonlinear fiber (HNLF) [1-4] or cascade second-order effects in periodicallypoled lithium niobate (PPLN) [5].

Moving to an integrated platform, which may increase the tolerance to the stimulated Brillouin scattering (SBS) compared to HNLFs and provide easier dispersion engineering and thus bandwidth, would be strongly beneficial for the future deployment of such a technology. A first attempt at providing nonlinearity compensation through OPC in a silicon waveguide was recently reported [6]. However, the impact of two-photon absorption (TPA) and consequent free carrier absorption (FCA) resulted in a poor conversion efficiency (CE) which limited the achievable optical signalto-noise ratio (OSRN) of the conjugate signal and, in turn, enabled increased nonlinear tolerance but no Q-factor improvement at the optimum launched power compared to straight transmission.

In this work, we demonstrate compensation of fiber Kerr nonlinearity through OPC using a silicon nano-rib waveguide with a lateral p-i-n diode as a nonlinear medium [7, 8]. The p-i-n diode enables suppression of FCA in the OPC module, which has previously limited the achievable performance improvements in demonstrations [6]. The nonlinearity compensation allows receiving 5-wavelength division multiplexed (WDM) 16-quadrature amplitude modulated (QAM) single-polarization channels with performance better than the hard-decision forward error correction (HD-FEC) threshold after 644-km dispersion-compensated transmission, performance not achievable in straight transmission.

\section{Samples characterization}

The silicon waveguide used in this work has been fabricated in the BiCMOS pilot line of IHP GmbH. The waveguides are manufactured on silicon-on-insulator (SOI) wafers with 220-nm top silicon layer and the $2 \mu \mathrm{m}$ buffer oxide, patterned and etched to provide nano-rib waveguide with a width of $500 \mathrm{~nm}$ and a slab thickness of $100 \mathrm{~nm}$. The waveguide is $4 \mathrm{~cm}$ long and has a lateral $\mathrm{p}-\mathrm{i}-\mathrm{n}$ diode with a separation of the $\mathrm{p}$ and $\mathrm{n}$ regions of $1.2 \mu \mathrm{m}$ [7]. The total linear insertion loss of the waveguide (fiber-to-fiber) is $13.4 \mathrm{~dB}$ for $40-\mathrm{V}$ reverse-bias applied to the diode, of which $4.8 \mathrm{~dB}$ are due to each of the fiber grating couplers, leading to a linear propagation loss of $0.96 \mathrm{~dB} / \mathrm{cm}$. The grating couplers are currently polarization sensitive, therefore limiting this demonstration to single-polarization signals. This might be overcome by using a coupling scheme that can accept both TE and TM polarizations [9]. The nonlinear properties of the waveguide are shown in Fig. 1(a) and 1(b) where the FWM input-to-output conversion efficiency (CE, defined as the power of the idler at the output vs. input signal power) is reported for a single continuous-wave $(\mathrm{CW})$ pump coupled into the waveguide together with a weak $\mathrm{CW}$ signal. The $\mathrm{CE}$ is measured for 
different pump powers (Fig. 1(a)) at a constant bias $(40 \mathrm{~V})$ and for different bias voltages at a constant pump power $(26 \mathrm{dBm})$ (Fig. 1(b)). The FWM bandwidth is approx. $10 \mathrm{~nm}$ and by increasing the pump power from $23 \mathrm{dBm}$ to 26 $\mathrm{dBm}$ (Fig.1(a)), the CE increases $3.7 \mathrm{~dB}$. On the other hand, by changing the bias voltage at 26 -dBm pump power, the CE improves by $5.9 \mathrm{~dB}$. The maximum obtained input-to-output $\mathrm{CE}$ was measured to be $-19.2 \mathrm{~dB}$.
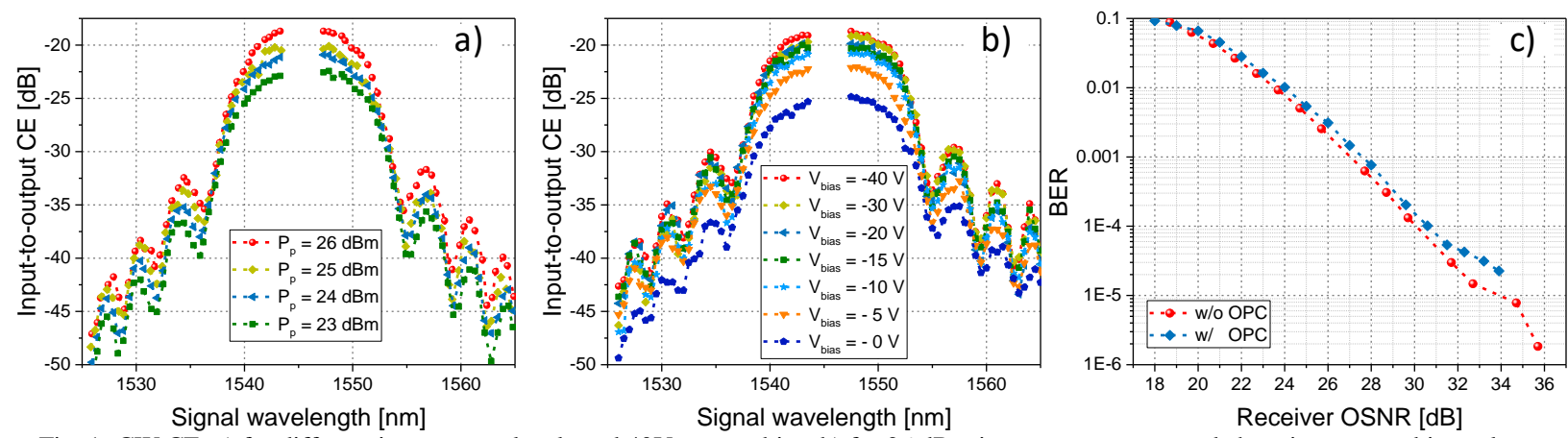

Fig. 1. CW CE: a) for different input power levels and $40 \mathrm{~V}$ reverse bias, b) for $26 \mathrm{dBm}$ input pump power and changing reverse bias voltage.

c) Back-to-back performance with and without OPC for the central channel of the 5-WDM 16-QAM signal band.

\section{System experiment}

The transmission testbed used to evaluate the performance of the OPC module based on the Si waveguide is shown in Fig. 2. The WDM 16-QAM transmitter is based on five 25-GHz spaced external cavity lasers (ECLs) modulated at 16-GBd in two IQ modulators driven by an arbitrary waveform generator (AWG) and individually decorrelated. The single-polarization WDM channels are polarization aligned and injected in a recirculating fiber loop. The transmission is based on three dispersion-compensated spans with erbium-doped fiber amplifiers (EDFAs) for a total loop length of $161 \mathrm{~km}$.

The OPC module based on the silicon waveguide is positioned within the loop and switches enable selecting either the OPC path or a parallel path consisting of an EDFA and a gain flattening filter (GFF). At the OPC stage, the WDM signals are amplified, band-pass filtered and vertically coupled together with a strong pump from an additional ECL into the Si waveguide. Pump and signals are polarization-aligned for maximum CE and their power levels have been optimized to minimize the penalty from the conversion in back-to-back (no transmission) conditions. The back-toback bit error ratio (BER) with and without OPC of the central WDM channel is shown in Fig. 1(c) as a function of the received OSNR varied by adding amplified spontaneous emission (ASE) noise at the receiver input. A pump power of $25 \mathrm{dBm}$ and 40-V reverse bias were sufficient to lead to an input-to-output CE of -19.5 dB (output-to-output CE of $-6 \mathrm{~dB}$, Fig. 3(a)) and the input signal power was set to $8.5 \mathrm{dBm}$ leading to a maximum receiver OSNR of $34 \mathrm{~dB}$ and an OSNR penalty below $0.5 \mathrm{~dB}$ (see Fig. 1(c)).

After a total of four loop turns $(644 \mathrm{~km})$, the WDM channels are sent to a single-polarization coherent receiver. The digitized signals are processed by off-line digital signal processing consisting of down-sampling, low-pass filtering, blind radius directed equalization (RDE), phase recovery and BER counting.

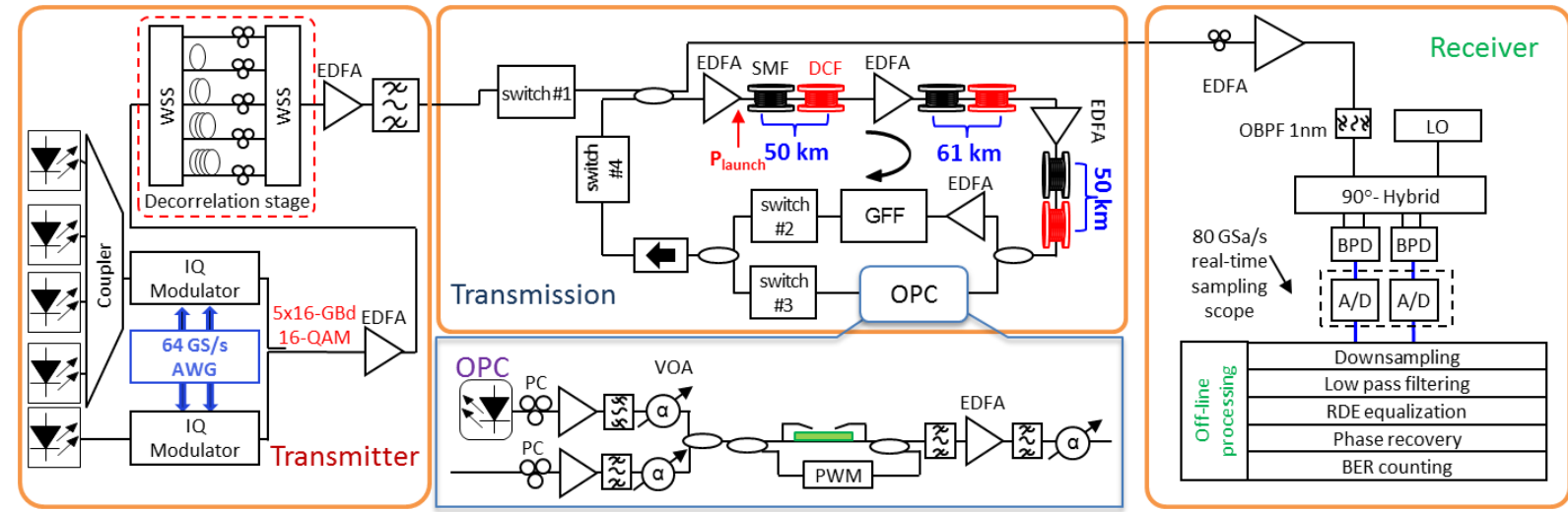

Fig. 2. System setup for the $5 \times 16-$ GBd 16-QAM modulated signal transmitted over 644 km long fiber optic transmission line with the silicon nano-rib waveguide based OPC module placed in the middle of transmission link.

\section{Results}


The nonlinearity compensation is evaluated by comparing the performance of the straight $644 \mathrm{~km}$ transmission to the performance obtained employing mid-link OPC, i.e., positioning the OPC stage at $322 \mathrm{~km}$. The Q-factor (derived from the BER) of the central WDM channel is shown in Fig. 3(b) as a function of the launched power (varied by the EDFAs at the input of each span) for both straight and OPC-based transmission. In the linear regime, no difference in performance is visible between straight and OPC-based transmission, confirming the low-penalty operation of the OPC already highlighted in Fig. 1(c). At the optimum launch power (increased by $2 \mathrm{~dB}$ for the OPC-based transmission), the presence of the OPC provides a 1-dB improvement in Q-factor. Additionally, (Fig. 3(c)) shows the performance of all the channels at optimum launch power $(-8 \mathrm{dBm}$ for straight and $-6 \mathrm{dBm}$ for OPC-based transmission). While only one channel $(194.66 \mathrm{THz})$ can achieve performance better than the HD-FEC threshold in straight transmission, the improvement provided by the OPC enables all of them to reach a Q-factor above the HDFEC threshold, thus proving the effectiveness of the Si waveguide based OPC module. The difference in performance among the channel is due to residual gain tilt in the transmission EDFAs.
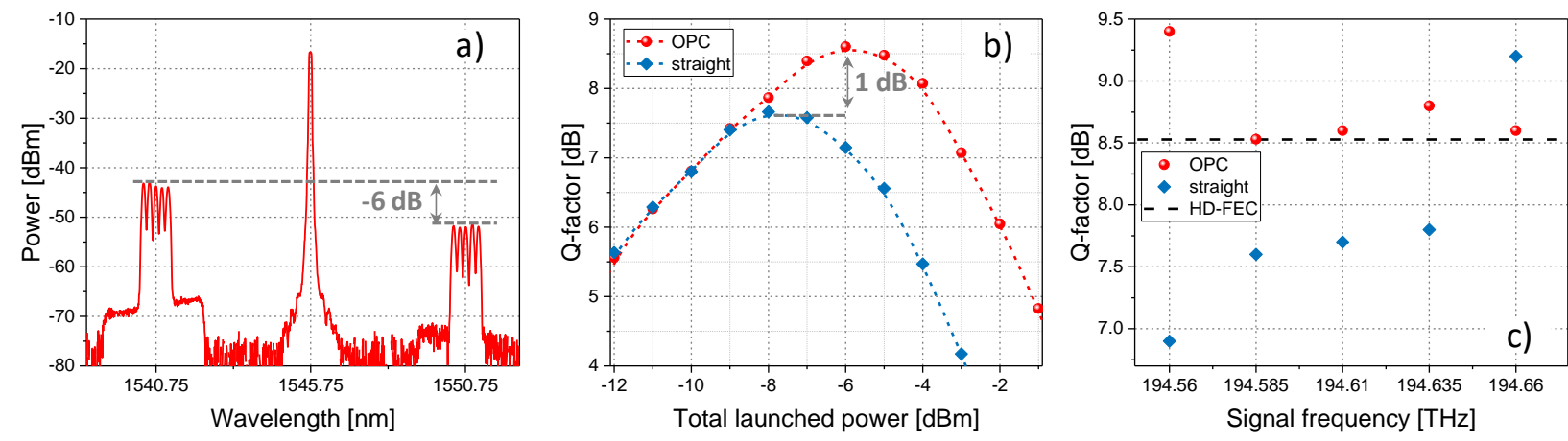

Fig. 3. a) Optical spectrum at the output of the waveguide. Q-factor with and without mid-link OPC after 644-km transmission for b) the central channel as a function of the launched power; and c) for all 5 WDM channels.

\section{Conclusions}

We presented an OPC module based on a silicon nano-rib waveguide with a reverse-biased lateral p-i-n diode. The decreased free carrier effects, owing to the diode, enabled a demonstration of high-quality optical phase conjugation using the silicon waveguide. Nonlinearity compensation provided by the OPC resulted in 1-dB Q-factor improvement in a 5×16-GBd 16-QAM dispersion compensated transmission over $644 \mathrm{~km}$. This Q factor enhancement allowed for receiving all five tested channels with a bit-error-ratio (BER) below HD-FEC.

\section{Acknowledgements}

This work was supported by the DFG projects SFB 787, ZI 1283/-1 and PE319/36.1, the DNRF CoE, SPOC (ref. DNRF123), and NANO-SPECs (DFF-4005-00558B).

\section{References}

[1] A.D. Ellis, et al., " $4 \mathrm{~Tb} / \mathrm{s}$ transmission reach enhancement using $10 \times 400 \mathrm{~Gb} / \mathrm{s}$ super-channels and polarization insensitive dual band optical phase conjugation," Journal of Lightwave Technology 34(8), 1717-1723 (2016).

[2] Y. Sun, et al., "Optical nonlinearity mitigation of $6 \times 10 \mathrm{GBd}$ polarization-division multiplexing 16-QAM signals in a field-installed transmission link," in Proc. OFC 2017, paper Th3J.2.

[3] K. Solis-Trapala, et al., "Doubled transmission reach for DP-64QAM signal over field-deployed legacy fiber systems enabled by MSSI," in Proc. ECOC 2015, paper Mo.3.6.2.

[4] I. Sackey, et al., "Kerr nonlinearity mitigation in 5×28-GBd PDM 16-QAM signal transmission over a dispersion-uncompensated link with backward-pumped distributed Raman amplification," Optics Express 22(22), 27381-27391 (2014).

[5] T. Umeki, et al., "Simultaneous nonlinearity mitigation in $92 \times 180-\mathrm{Gbit} / \mathrm{s}$ PDM-16QAM transmission over 3840 km using PPLN-based guardband-less optical phase conjugation," Optics Express 24(15), 16945-16951 (2016).

[6] D. Vukovic, et al., "Multichannel nonlinear distortion compensation using optical phase conjugation in a silicon nanowire," Optics Express 23(3), 3640-3646 (2015).

[7] A. Gajda, et al., "Highly efficient CW parametric conversion at $1550 \mathrm{~nm}$ in SOI waveguides by reverse biased p-i-n junction," Optics Express 20(12), 13100-13107 (2012).

[8] I. Sackey, et al. "1.024 Tb/s wavelength conversion in a silicon waveguide with reverse-biased p-i-n junction," Optics Express 25(18), 21229-21240 (2017)

[9] F. Van Laere, et al., "Focusing Polarization Diversity Grating Couplers in Silicon-on-Insulator," Journal of Lightwave Technology 27(5), 612618 (2009). 